LÓPEZ CAMELO, A.F.; GÓMEZ, P.A. Modelling postharvest colour changes in long shelf life tomatoes. In: $X X V$ INTERNATIONAL HORTICULTURAL CONGRESS. 1998, Bruselas. Abstracts... Bruselas: Bélgica, 1998. Supplement, p. 9.

MALUNDO, T.M., SHEWFELT, R.L.; SCOTT, J.W. Flavor quality of fresh tomato (Lycopersicum esculentum Mill.) as affected by sugar and acid levels. Postharvest Biology and Technology, v. 6, 103-110, 1995.

PEPPELENBOS, H.W. Gas exchange models and prediction of disorders in fruits. Workshop on Food Quality Modelling. Katholieke Universiteit Leuven. Belgium, 1997.

PEIRIS, K., DULL, G., LEFFLER, R.; KAYS, S. Near infrared spectrometric technique for nondestructive determination of soluble solids content in processing tomatoes. Journal of the American Society for Horticultural Science, v, 123, p. 1089-1093, 1998.
SALTVEIT, M. Effect of ethylene on quality of fresh fruits and vegetables. Postharvest Biology and Technology, n. 15, p. 279-292, 1999.

SALUNKE, D.K.; WU, M.T. Effects of low oxygen atmosphere storage on ripening and associated biochemical changes of tomato fruits. Journal of the American Society for Horticultural Science, v. 98, n. 1, p. 12-14, Jan. 1973.

SALUNKE, D.; DESAI, B. Postharvest biotechnology of fruits. Florida: CRC Press, 1984, $18 \mathrm{p}$.

SHEWFELT, R.; PRUSSIA, S. Postharvest handling. A system approach. California: Academic Press, 1993. 358 p.

SILVA, F., CHAU, K., BRECHT, J.; SARGENT, $\mathrm{S}$. Modified atmosphere packaging for mixed loads of horticultural commodities exposed to two postharvest temperatures. Postharvest Biology and Technology, v. 17, p. 1-9, 1999.
TIJSKENS, L.M.; EVELO, R.G. Modelling color of tomatoes during postharvest storage. Postharvest Biology and Technology, v. 4, p. 8598, 1994.

THOMPSON, A. Controlled atmosphere storage of fruits and vegetables. London: CAB International, 1998. 278 p.

TUCKER, G., ROBERTSON, N.; GRIERSON, D. Changes in polygalacturonase isoenzymes during the ripening of normal and mutant tomato fruit. European Journal of Biochemestry, v. 112, p. 119-124, 1980.

USDA. Visual aid. The California Tomato Board. 1975.

WILLS, R.; LEE, T.; GRAHAM, D.; McGLASSON, W.; HALL, E. Postharvest. An introduction to the physiology and handling of fruit and vegetables. Kensington, Australia. New South Wales University Press. 1981. 161 p.

YANG, C.; CHINAN, M. Modeling the effect of $\mathrm{O}_{2}$ and $\mathrm{CO}_{2}$ on respiration and quality of stored tomatoes. Transactions of ASAE, v. 31, n. 3, p. 920-925, 1988

DAROS, M.; AMARAL JÚNIOR, A.T.; PEREIRA, T.N.S.; LEAL, N.R.; FREITAS, S.P.; SEDIYAMA, T. Caracterização morfológica de acessos de batatadoce. Horticultura Brasileira, Brasília, v. 20, n. 1, p. 43-47, março 2002.

\title{
Caracterização morfológica de acessos de batata-doce ${ }^{1}$.
}

\author{
Máskio Daros $^{1 /}$; Antônio T. Amaral Júnior ${ }^{1 /}$; Telma Nair S. Pereira ${ }^{1 /}$; Nilton R. Leal ${ }^{1 /}$; Silvério P. Freitas ${ }^{1 /}$; \\ Tocio Sediyama ${ }^{2 /}$ \\ ${ }^{1 / U E N F-C C T A, ~ A v . ~ A l b e r t o ~ L a m e g o ~ 2000, ~ H o r t o, ~ 28.015-620 ~ C a m p o s ~ d o s ~ G o y t a c a z e s ~-~ R J ; ~}{ }^{2 / U F V, ~ 36.571-000 ~ V i c ̧ o s a ~-~ M G ; ~}$
}

E-mail: daros@uenf.br

\section{RESUMO}

Objetivando caracterizar morfologicamente acessos de batatadoce da Coleção de Germoplasma da UENF e visando otimizar a utilização de genomas de interesse para o melhoramento, foram instalados dois experimentos, ambos em blocos ao acaso com três repetições. Os plantios foram realizados em novembro e em dezembro de 1997, ambos na cidade de Campos dos Goytacazes (RJ). Para tanto, quatorze acessos de batata-doce foram avaliados quanto a descritores da parte aérea e das raízes, num total de vinte características. Constatou-se a ocorrência de variabilidade genética entre os acessos, proporcionada principalmente pelas características pubescência do ápice das ramas, pigmentação das nervuras inferiores da folha e formato das raízes. Os acessos 'Amarelinha', 'Roxinha', 'WON-B' e 'Campina 3' apresentaram características de interesse para o mercado consumidor Norte- Fluminense.

Palavras-chave: Ipomoea batatas (L.) Lam., descrição morfoagronômica, descritores, recursos genéticos.

\section{ABSTRACT}

\section{Morphologic characterization of sweet potato.}

To characterize morphologically accesses of sweet potato from the germplasm collection of the Universidade Estadual do Norte Fluminense (UENF) Brazil, as a way to optimize the utilization of important genomes for plant breeding, two experiments were carried out in field conditions, both in a randomized blocks design with three replications. The experiments were done in November at UENF and December in Campos. For such experiment, fourteen sweet potato accesses were evaluated for different traits related to aerial parts, and root descriptors, in a total of twenty characteristics. Genetic variability between accesses was observed, mainly due to characteristics of pubescence of the stem apex, pigmentation of the inferior veins of leaves and shape of roots. The accesses, Amarelinha, Roxinha, WON-B and Campina 3 presented interesting characteristics for the market of north-fluminense region.

Keywords: Ipomoea batatas (L.) Lam., morphoagronomic description, descriptors, genetic resources.

\section{(Aceito para publicação em 3 de dezembro de 2.001)}

\begin{abstract}
A espécie Ipomoea batatas é cultivada em todo território nacional, apresentando uma infinidade de formas, com grande diversidade fenotípica e genotípica. Praticamente todos os Estados brasileiros possuem cultivares peculiares,
\end{abstract}

porém, muito do que se conhece é mera duplicata, visto ocorrerem diversas cultivares iguais com o mesmo nome e viceversa (Miranda, 1982; Murilo, 1990).

Por conseguinte, a caracterização morfológica constitui em tarefa de gran- de importância para a cultura da batatadoce, para evitar o plantio de formas genômicas semelhantes e o conseqüente estreitamento genético da espécie.

A caracterização morfológica consiste em fornecer uma identidade para

\footnotetext{
${ }^{1}$ Parte da tese de mestrado defendida pelo primeiro autor em outubro de 1999 na UENF.
} 
cada entrada através do conhecimento de uma série de dados que permitam estudar a variabilidade genética de cada amostra (Ramos \& Queiroz, 1999).

Considerando a necessidade de um estudo sobre a recomendação de cultivares para o Município de Campos dos Goytacazes RJ., e de se conhecer, valorizar e utilizar os acessos da coleção de I. batatas, desenvolveu-se este trabalho com o objetivo de diferenciar os acessos da Coleção de germoplasma da UENF e, com isso, indicar formas promissoras para o melhoramento genético e para o atendimento ao mercado consumidor.

\section{MATERIAL E MÉTODOS}

A caracterização morfológica foi realizada na Unidade de Apoio à Pesquisa do Centro de Ciências e Tecnologias Agropecuárias da Universidade Estadual do Norte Fluminense e na Estação Experimental de Campos da PESAGRO-RIO, no Município de Campos dos Goytacazes, onde foram instalados experimentos no campo em novembro e dezembro de 1997, respectivamente.

O clima da região é classificado como tropical chuvoso, clima de bosque (AM), segundo Koeppen, citado por Ometo (1981), com uma precipitação média anual de $1023 \mathrm{~mm}$. O primeiro experimento foi implantado em solo fúlvico de textura argilosa com 2,2\% de matéria orgânica e o segundo em latossolo amarelo com a mesma textura e $0,14 \%$ de matéria orgânica.

Utilizaram-se quatorze acessos de $I$. batatas, dois dos quais, provenientes de olericultores do próprio município (Roxinha e Amarelinha). Dois foram oriundos de Itaguaí (WON-A e WON-B) e um de Magé (Rosinha de Verdan), três do Estado da Paraíba (Paraíba, Mandioca e Campina 3) e os demais (Acesso 07, Talo roxo, Linha 5, Rosada, Roxa-roxa e Mazomba) sem procedência conhecida.

O delineamento experimental utilizado foi blocos casualizados com quatorze tratamentos e três repetições. Cada bloco foi composto por duas leiras de quatorze metros de comprimento e 0,50 $\mathrm{m}$ de altura, com espaçamento de $0,25 \mathrm{~m}$ entre plantas e 1,5 m entre o topo das leiras. As áreas experimentais foram submetidas a uma aração e uma gradagem, feitas, respectivamente, a um mês e uma semana antes do plantio; posteriormente, foram feitas leiras no terreno.

As avaliações da parte vegetativa foram feitas três meses após o plantio e as da raiz foram realizadas por ocasião da colheita (120 dias após o plantio).

As adubações foram feitas com base na análise de solo. De acordo com os valores obtidos nesta análise, fez-se as seguintes adubações: no ambiente 1 (UENF-CCTA) aplicou-se $10 \mathrm{~kg} / \mathrm{ha}$ de nitrogênio, $180 \mathrm{~kg} /$ ha de $\mathrm{P}_{2} \mathrm{O}_{5}$ e $30 \mathrm{~kg} /$ ha de $\mathrm{K}_{2} \mathrm{O}$. No ambiente 2 (PESAGRORIO), aplicou-se a mesma quantidade de nitrogênio, metade da dose de fósforo e o dobro da dose de potássio.

O nitrogênio (sulfato de amônio) foi aplicado aos quinze dias (dose única), juntamente com o cloreto de potássio. O fósforo (superfosfato simples) foi aplicado por ocasião do plantio.

As irrigações por aspersão compreenderam duas aplicações semanais até três semanas após o plantio e uma irrigação semanal desta data até a colheita. $O$ controle de invasoras foi feito de acordo com a necessidade.

A caracterização morfológica foi realizada com o material em pleno desenvolvimento vegetativo, segundo proposto por Huamán (1996), exceto para características referentes às raízes tuberosas, que seguiram o padrão de caracterização recomendado pelo ' 'International Board for Plant Genetic Resources" (IBPGR) (1991). Os dados referentes às características de folhas $\mathrm{e}$ de pecíolos foram obtidos da parte central das ramas (folhas maduras), utilizando-se três folhas por planta e quatro plantas por parcela dentro de um bloco em cada experimento. Os dados referentes às raízes foram obtidos de todo material colhido de seis plantas úteis de cada parcela.

Foram avaliados, conforme preconizado por Huamán (1996) e IBPGR (1991): a) tamanho da folha (TF) [pequena $(<8 \mathrm{~cm})$, média $(8-15 \mathrm{~cm})$, grande $(16-25 \mathrm{~cm})$ ou muito grande $(>25$ $\mathrm{cm})$ ], com notas $3 ; 5 ; 7$, ou 9 , respectivamente; b) perfil geral da folha (PG) (arredondada, reniforme, cordada, triangular, lanceolada, lobulada ou quase di- vidida), com notas $1 ; 2 ; 3 ; 4 ; 5 ; 6$ ou 7 , respectivamente; c) tipo de lóbulo da folha (TL) (ausência de lóbulos, lóbulos muito superficiais, lóbulos superficiais, moderados, profundos ou muito profundos), com notas $0 ; 1 ; 3 ; 5 ; 7$ ou 9 , respectivamente; d) número de lóbulos da folha (NL), obtido pela contagem dos lóbulos de cada folha, descartando-se os basais; e) forma do lóbulo central da folha (FLC) (ausente, dentada, triangular, semi circular; semi elíptica, elíptica, lanceolada, oblongolanceolada, linear ou linear-estreito), com notas o; $1 ; 2 ; 3$; $4 ; 5 ; 6 ; 7 ; 8$ ou 9 , respectivamente; f) cor da folha madura (FM) (amarelo-verde, verde, verde com bordas roxas, verde cinzento, verde com nervuras roxas na face, ligeiramente roxa, predominantemente roxa, verde na face superior e roxo na inferior ou roxo em ambas as superfícies), com notas $1 ; 2 ; 3 ; 4 ; 5 ; 6$; $7 ; 8$ ou 9 , respectivamente; g) cor da folha imatura (FI) (amarelo-verde, verde, verde com bordas roxas, verde cinzento, verde com nervuras roxas na face, ligeiramente roxa, predominantemente roxa, verde na face superior e roxo na inferior ou roxo em ambas as superfícies), com notas $1 ; 2 ; 3 ; 4 ; 5 ; 6 ; 7 ; 8$ ou 9 , respectivamente; h) pigmentação do pecíolo (PIG) (verde, verde com roxo próximo ao talo, verde com roxo próximo à folha, verde com roxo em ambos os extremos, verde com manchas roxas ao longo do pecíolo, verde com bandas roxas, roxo com verde próximo à folha, alguns verdes outros roxos ou totalmente roxo), com notas $1 ; 2 ; 3 ; 4 ; 5 ; 6 ; 7 ; 8$ ou 9 , respectivamente; i) comprimento do pecíolo (COM) [muito curto $(<10 \mathrm{~cm})$, curto $(10-20 \mathrm{~cm})$, intermediário $(21-30 \mathrm{~cm})$, longo $(31-40 \mathrm{~cm})$ ou muito longo ( $>40 \mathrm{~cm})$ ], com notas $1 ; 3$; 5 ; 7 ou 9 , respectivamente; j) pigmentação das nervuras inferiores (PNI) (amarelo, verde, mancha roxa na base da nervura principal, mancha roxa em várias nervuras ou nervura principal parcialmente roxa, nervura principal predominantemente ou totalmente roxa, todas nervuras parcialmente roxas, todas as nervuras predominantemente ou totalmente roxas, toda face inferior ou nervuras totalmente roxas), com notas $1 ; 2 ; 3 ; 4 ; 5 ; 6 ; 7 ; 8$ ou 9 , respectivamente; 1) coloração predominante das ramas $(\mathrm{CP})$ (verde, verde com poucas 
Tabela 1. Caracterização de alguns descritores referentes à parte aérea de quatorze acessos de batata-doce para as condições ambientais do Município de Campos dos Goytacazes. Campos dos Goytacazes, UENF, 1997.

\begin{tabular}{|c|c|c|c|c|c|c|c|c|c|c|c|c|c|}
\hline \multirow{3}{*}{ Acesso } & \multicolumn{3}{|c|}{ Ramas } & \multicolumn{5}{|c|}{ Folha madura } & \multirow{3}{*}{ PNI } & \multicolumn{2}{|c|}{$\begin{array}{l}\text { Cor da } \\
\text { folha }\end{array}$} & \multicolumn{2}{|c|}{ Pecíolo } \\
\hline & \multicolumn{2}{|c|}{ PIG } & \multirow[t]{2}{*}{ PAR } & \multicolumn{4}{|c|}{ Forma } & \multirow{2}{*}{ Tamanho } & & \multirow{2}{*}{ FM } & \multirow{2}{*}{$\mathbf{F I}$} & \multirow{2}{*}{ PIG } & \multirow{2}{*}{ COM } \\
\hline & CP & CS & & PG & TL & $\mathbf{N L}$ & FLC & & & & & & \\
\hline Roxinha & 3 & 0 & 9 & 6 & 5 & 5 & 4 & $9-13(5)$ & 8 & 2 & 3 & 4 & 5 \\
\hline Amarelinha & 1 & 2 & 5 & 6 & 7 & 7 & 9 & $8-10(5)$ & 4 & 2 & 2 & 3 & 3 \\
\hline Acesso 7 & 9 & 2 & 3 & 6 & 3 & 5 & 4 & $10-14(5)$ & 9 & 9 & 9 & 9 & 5 \\
\hline Talo roxo & 1 & 0 & 3 & 6 & 3 & 5 & 2 & $11-14(5)$ & 5 & 2 & 3 & 4 & 7 \\
\hline Linha 5 & 1 & 0 & 3 & 6 & 3 & 9 & 1 & $10-12(5)$ & 7 & 2 & 2 & 4 & 5 \\
\hline WON-A & 1 & 0 & 0 & 6 & 3 & 9 & 1 & $10-15(5)$ & 5 & 2 & 2 & 4 & 7 \\
\hline Paraíba & 1 & 2 & 0 & 3 & 1 & 1 & 1 & $8-10(5)$ & 5 & 2 & 3 & 1 & 5 \\
\hline Mandioca & 3 & 6 & 3 & 6 & 3 & 5 & 2 & $10-15(5)$ & 7 & 2 & 2 & 3 & 7 \\
\hline WON-B & 1 & 2 & 3 & 6 & 3 & 5 & 4 & $10-12(5)$ & 7 & 2 & 3 & 5 & 7 \\
\hline Campina 3 & 1 & 2 & 3 & 6 & 3 & 5 & 2 & $11-14(5)$ & 7 & 2 & 2 & 3 & 7 \\
\hline Rosada & 1 & 2 & 5 & 6 & 3 & 5 & 2 & $10-13(5)$ & 2 & 2 & 3 & 1 & 5 \\
\hline Roxa-roxa & 1 & 7 & 7 & 6 & 1 & 5 & 2 & $11-15(5)$ & 7 & 2 & 4 & 1 & 5 \\
\hline R de Verdan & 1 & 3 & 7 & 6 & 5 & 5 & 4 & $10-15(5)$ & 7 & 2 & 3 & 3 & 5 \\
\hline Mazomba & 1 & 5 & 7 & 6 & 3 & 5 & 2 & $10-14(5)$ & 2 & 2 & $4 / 9$ & 1 & 3 \\
\hline
\end{tabular}

PIG = pigmentação da rama; $\mathrm{CP}=$ cor predominante da rama $(1=$ verde, $3=$ verde com poucas manchas rosadas, $9=$ totalmente roxo escuro); $\mathrm{CS}=$ cor secundária da rama $(0=$ ausente, $2=$ ápice verde, $3=$ nós verdes, 5 = ápice rosado, $6=$ nós rosados, $7=$ outros $) ; \mathrm{PAR}=$ pubescência do ápice da rama $(0=$ nenhuma, $3=$ rala, $5=$ moderada, $7=$ densa, $9=$ muito densa $) ; P G=$ perfil geral da folha madura $(3=$ cordada, $6=$ lobulada); $\mathrm{TL}=$ tipo de lóbulo da folha madura $(1=$ lóbulos muito superficiais, $3=$ superficiais, $5=$ moderados, $7=$ profundos $)$; $\mathrm{NL}$ = número de lóbulos da folha madura; FLC = forma do lóbulo central da folha madura $(1=$ dentada, 2 = triangular, $4=$ semi-elíptica, 9 $=$ linear-estreito); TAM $=$ tamanho da folha $(5=$ folha mediana); $\mathrm{PNI}=$ pigmentação das nervuras inferiores $(2=$ verde, $4=$ manchas roxas em várias nervuras, $5=$ nervura principal parcialmente roxa, $7=$ todas as nervuras parcialmente roxas, $8=$ totalmente roxas, $9=$ toda face inferior e nervuras totalmente roxas); $\mathrm{FM}=$ cor da folha madura $(2=$ verde, $9=$ roxa em ambas superfícies $) ; \mathrm{FI}=$ cor da folha imatura $(2=$ verde, $3=$ verde com bordo roxo, $4=$ verde cinzento, $9=$ roxa em ambas superfícies $) ; \mathrm{PIG}=$ pigmentação do pecíolo $(1=$ verde, $3=$ verde com roxo próximo a folha, $4=$ verde com roxo em ambos extremos, $5=$ verde com manchas roxas ao longo do pecíolo, $9=$ totalmente roxo); $\mathrm{COM}=$ comprimento do pecíolo $(1=$ muito curto, $3=$ curto, 5 = intermediário $) .{ }^{*}$ Rosinha de Verdan

manchas rosadas, verde com muitas manchas rosadas, verde com muitas manchas rosadas escuras, predominantemente rosado, predominantemente rosado escuro, totalmente rosado ou totalmente rosado escuro), com notas $1 ; 3 ; 4 ; 5 ; 6 ; 7 ; 8$ ou 9 , respectivamente); m) cor secundária das ramas (CS) (ausente, base verde, ápice verde, nós verdes, base rosada, ápice rosado, nós rosados ou outros), com notas $0 ; 1 ; 2 ; 3 ; 4 ; 5 ; 6$ ou 7 , respectivamente; n) pubescência do ápice das ramas (PAR) (ausência de pilosidade, pilosidade rala, moderada, densa ou muito densa), com notas $0 ; 3 ; 5 ; 7$ ou 9 , respectivamente; o) formato da raiz (FOR) [redondo (perfil quase circular com uma proporção de largura e comprimento de 1:1), redondo elíptico (perfil ligeiramente circular com bordos agudos e uma proporção de largura e comprimento de 2:1), elíptico (perfil com a largura máxima igual à distância de ambos os extremos e uma proporção de largura e comprimento não superior a 3:1), ovado (perfil semelhante a um ovo), obovado (perfil inverso ao ovado), oblongo (perfil quase retangular com os lados paralelos), largo oblongo (perfil oblongo com uma proporção de largura e comprimento superior a 3:1), largo elíptico (perfil elíptico com uma proporção de largura e comprimento superior a 3:1) ou largo irregular ou curvado, com notas $1 ; 2 ; 3 ; 4 ; 5 ; 6$; $7 ; 8$ ou 9 , respectivamente; p) defeitos na superfície da raiz (DS) (ausente, periderme com pele de crocodilo, veias proeminentes, constrições horizontais superficiais, constrições horizontais profundas, fendas longitudinais superficiais, fendas longitudinais profundas, constrições e rachaduras profundas ou outros), com notas $0 ; 1 ; 2 ; 3 ; 4 ; 5 ; 6 ; 7$ ou 8 , respectivamente; q) espessura do córtex (ESPCORT) [muito fina (1 $1 \mathrm{~mm} \mathrm{ou}$ menos), fina (1-2 mm), intermediária (2$3 \mathrm{~mm}$ ), grossa (3-4 mm), muito grossa ( $>4 \mathrm{~mm}$ )], com notas $1 ; 3 ; 5 ; 7$ ou 9 , respectivamente; r) cor predominante da periderme (CORPRED) (branco, creme, amarelo, alaranjado, marrom-alaranjado, rosado, roxo, roxo-avermelhado ou vermelho escuro), com notas $1 ; 2 ; 3 ; 4 ; 5 ; 6$; 7; 8 ou 9 , respectivamente; s) intensidade da cor (INT) (pálida, intermediária ou escura), com notas 1,2 ou 3 , respectivamente; t) cor secundaria da periderme (CORSEC) (classifica-se nos mesmos tons que a cor predominante); $\mathrm{u}$ ) cor predominante da polpa (CPP) (branca, creme, creme-escuro, amarelo-pálido, amarelo-escuro, alaranjado-pálido, alaranjado-intermediário, alaranjado-escuro ou fortemente pigmentado com antocianinas), com notas $1 ; 2 ; 3 ; 4 ; 5 ; 6$; $7 ; 8$ ou 9 , respectivamente. 
Tabela 2. Caracterizações de alguns descritores referentes à raiz de quatorze acessos de batata-doce avaliados para as condições do Município de Campos dos Goytacazes. Campos dos Goytacazes, UENF, 1997.

\begin{tabular}{lccccccc}
\hline \multirow{2}{*}{ Acesso } & \multicolumn{8}{c}{ Raíz } \\
\cline { 2 - 8 } & \multirow{2}{*}{ FOR } & DS & ESPCOR- & \multicolumn{3}{c}{ Cor da periderme } & \multicolumn{3}{c}{ Cor da polpa } \\
\cline { 5 - 8 } & & & T & CORPRED & INT & COR1SEC & CPP \\
\hline Roxinha & $8 / 7$ & 2 & 7 & 6 & 1 & 1 & 1 \\
Amarelinha & $8 / 9$ & 3 & 3 & 6 & 1 & 1 & 1 \\
Acesso 7 & 8 & 3 & 9 & 3 & 2 & 3 & 4 \\
Talo roxo & 5 & 8 & 9 & 4 & 2 & 1 & 4 \\
Linha 5 & $2 / 3$ & 8 & 7 & 3 & 2 & 1 & 2 \\
WON-A & $9 / 8$ & 3 & 7 & 3 & 2 & 0 & 2 \\
Paraíba & 1 & 2 & 9 & 6 & 3 & 1 & 7 \\
Mandioca & 3 & 0 & 9 & 6 & 2 & 0 & 2 \\
WON-B & $3 / 5$ & 1 & 7 & 5 & 1 & 1 & 3 \\
Campina 3 & $7 / 4$ & 5 & 5 & 4 & 2 & 2 & 2 \\
Rosada & $8 / 9$ & 8 & $7 / 4$ & 6 & 2 & 1 & 2 \\
Roxa roxa & $5 / 6$ & 0 & 3 & 9 & 3 & 9 & 9 \\
Rosinha de Verdan & 7 & 2 & 9 & 6 & 1 & 1 & 2 \\
Mazomba & $2 / 3$ & 2 & 5 & 6 & 1 & 1 & 2 \\
\hline
\end{tabular}

$\mathrm{FOR}=$ formato da raiz $[1=$ redondo $(\mathrm{L} / \mathrm{A} 1: 1), 2=$ redondo-elíptico $(\mathrm{L} / \mathrm{A}<2: 1), 3=$ elíptico $(\mathrm{L} / \mathrm{A}<3: 1), 4=$ ovado, $5=$ obovado, $6=$ oblongo $(\mathrm{L} / \mathrm{A}=2: 1), 7$ = largo oblongo $(\mathrm{L} / \mathrm{A}>3: 1), 8=$ largo elíptico $(\mathrm{L} / \mathrm{A}>3: 1), 9$ = largo irregular ou curvado]; $\mathrm{DS}=$ defeito da superfície [ $0=$ ausente, $1=$ pele de crocodilo, $2=$ veias proeminentes, $3=$ constrições horizontais superficiais, $5=$ fendas longitudinais superficiais, $8=$ outros]; ESPCORT $=$ espessura do córtex [ $3=$ fina $(1-2 \mathrm{~mm}), 5=$ intermediária $(2-3 \mathrm{~mm}), 7=$ grossa $(3-4 \mathrm{~mm}), 9=$ muito grossa $(>4 \mathrm{~mm})]$; CORPRED $=$ cor predominante da periderme $[3=$ amarela, $4=$ alaranjada, $5=$ marrom alaranjada, $6=$ rosada, $7=$ roxa $] ;$ INT $=$ intensidade da cor da periderme[ 1 = pálida, $2=$ intermediária, $3=$ escura]; CORSEC $=$ cor secundária da periderme $[0=$ ausente, $1=$ branca, $2=$ creme, $3=$ amarela, $7=$ roxa]; $\mathrm{CPP}=$ cor predominante de polpa $[1=$ branca, $2=$ creme, $3=$ creme escuro, $4=$ amarelo pálido, $7=$ alaranjado intermediário, $9=$ fortemente pigmentado com antocianinas $]$.

\section{RESULTADOS E DISCUSSÃo}

Observa-se, que os descritores referentes à parte aérea revelaram elevada variabilidade genética entre os acessos inclusos nos experimentos (Tabela 1). O perfil geral e a cor da folha madura foram as características que menos proporcionaram variabilidade entre os acessos, o que se opõe à informação dada por Murilo (1990) onde é citado o formato das folhas como uma das características mais importantes na distinção genotípica. Por outro lado, a cor secundária das ramas e a pigmentação das nervuras inferiores da folha foram as que apresentaram maior variação. A pubescência do ápice das ramas foi, de todas as características avaliadas, a única que apresentou todas as classes possíveis para os quatorze acessos avaliados. No que se refere à cor predominante da rama a maioria dos acessos (80\%) se encontraram dentro da mesma classe. Quanto à cor secundária, os acessos foram distribuídos de forma mais diferen- ciada. Portanto, a cor secundária da rama possibilitou maior discernimento dos acessos. De forma análoga, a pubescência do ápice das ramas apresentou-se como um bom descritor para a caracterização. A característica pigmentação das nervuras inferiores da folha possibilitou boa discriminação dos acessos, tornando-se uma boa indicadora da variabilidade presente, com a vantagem de ser de fácil classificação. Fica evidente, ao se comparar a cor da folhagem madura com a cor da folhagem imatura (Tabela 1), que a última se destacou pela maior variação para os acessos avaliados, revelando-se de maior utilidade na distinção genotípica. Quanto ao pecíolo, a pigmentação apresentou maior número de classes do que o comprimento. Para o comprimento do pecíolo, das cinco classes formadas, duas (números sete e nove) não ocorreram na avaliação.

Na Tabela 2 são apresentados alguns descritores referentes à raiz dos quatorze acessos de batata-doce avaliados. Per- cebe-se pelo mesmo, no que se refere às raízes tuberosas, que o formato foi a característica mais variável, com grande diversidade entre os acessos, inclusive dentro destas. Esta característica apresentou todas as classes possíveis de discriminação concordando com Vasconcellos (1986), que cita o formato das raízes como característica muito importante na descrição de cultivares. Cinco dos acessos avaliados (Roxinha, Amarelinha, Acesso 7, WON-A e Rosada) apresentaram o formato largoelíptico para raízes, considerado ideal para o comércio. Com exceção do acesso 7 , houve alguma variação, porém com menor expressão. Com o formato elíptico, também desejável ao comércio, cita-se Linha 5, Mandioca, WON-B e Mazomba ocorrendo também alguma variação como a característica anterior.

Outra característica de fácil identificação e que permite boa diferenciação é a presença de defeitos na superfície da raiz. Apesar de não terem ocorrido duas das classes, foi possível a diferen- 
ciação entre os acessos. Não se levando em conta a parte aérea, os defeitos na superfície da raiz diferem um acesso de outro, mesmo que tenham formato, cor e produção iguais. Entre estes, as nervuras proeminentes foram as que mais se destacaram, ocorrendo em quatro cultivares avaliadas (Roxinha, Paraíba, Rosinha de Verdan e Mazomba), seguidas pelas constrições horizontais superficiais, que ocorreram nas cultivares Amarelinha, Acesso $7 \mathrm{e}$ WON-A (Tabela2). De todos os acessos avaliados, apenas Roxa-roxa e Mandioca não apresentaram qualquer tipo de defeito na superfície da raiz; entretanto a cultivar Roxa-roxa possui uma coloração arroxeada na periderme e polpa não aceitável para o comércio local (Tabela 1). Ao contrário da característica anterior, a espessura do córtex não é de fácil obtenção, apresentando pequeno número de classes e podendo estar sujeito a erros de medições. A cor da periderme é outra característica tida como uma das mais importantes na diferenciação. Sua importância vai além do aspecto morfológico, estando mais relacionada com o aspecto comercial, onde há uma restrição de matizes preferidas pelo consumidor (creme, marromalaranjado e rosada). Para o trabalho em questão, a coloração rosada, que é aceitável pelo mercado, esteve em 50\% dos acessos (Roxinha, Amarelinha, Paraíba, Mandioca, Rosada, Rosinha de Verdan e Mazomba) (Tabela 2). Todavia, não se deve desconsiderar a cultivar WON$\mathrm{B}$, por apresentar cor marrom-alaranjada para essa característica, que também é de boa aceitabilidade pelo comércio. A cor secundária da periderme não proporcionou boa diferenciação, visto que 9 dos 14 acessos tiveram predominância do matiz branco. Semelhante ao que ocorreu com a característica cor secundária da periderme, a cor predominante de polpa também variou pouco entre os acessos estudados, com a maioria deles dentro de uma mesma classe, que é aceitável pelo comércio. Vale ressaltar que tais características são suficientes para diferenciação, porém, no presente trabalho houve uma certa sobreposição das mesmas.

É importante destacar também que embora as características comerciais sejam definidas pelo sistema radicular, a parte aérea revelou maior variabilidade, sendo mais útil na discriminação dos acessos.

Pode-se concluir que os genótipos 'Roxinha' e 'WON-B' são promissores para futuros programas de melhoramento, visto atenderem às exigências do mercado consumidor.

\section{LITERATURA CITADA}

CAMARGO, A.P. Observações preliminares sobre o ciclo vegetativo da batata-doce. Bragantia, Campinas, v. 5, p. 797-821, 1945.

HUAMÁN, Z. Botânica sistemática y morfológica de la planta de batata o camote. In: Manual de manejo de germoplasma de batata o camote (Ipomoea batatas). Lima: CIP, 1996. p. 01-16. IBPGR. Descriptores de la batata. Rome, 1991. $134 \mathrm{p}$.

MIRANDA, J.E.C. Batata-doce. Evolução e melhoramento. Piracicaba: USP/ESALQ, 1982. 139 p. (Monografia graduação).

MURILO, D.V. Cultivares de batata-doce. In: ENCONTRO DE PROFESSORES, PESQUISADORES E EXTENSIONISTAS DO RIO GRANDE DO NORTE, 4., 1990, Mossoró. Anais...Mossoró: Escola Superior de Agricultura de Mossoró - ESAM, 1990. p. 27-29.

OMETTO, J.C. Bioclimatologia tropical. São Paulo: Agronômica Ceres, 1981. p. 390-398

RAMOS, S.R.R. ; QUEIROZ, M.A. Caracterização morfológica: experiência do BAG de cucurbitáceas da Embrapa Semi - Árido, com acessos de abóbora e moranga. Horticultura brasileira, Brasília, v. 17, suplemento, p. 9-12, 1999.

VASCONCELLOS, H.O.; LEAL, N.R.; ALMEIDA, D.L.; CARVALHO, M.P.M. Caracterização de germoplasma de batata-doce (Ipomoea batatas (Lam.)) da Estação Experimental de Itaguaí. Itaguaí: Pesagro-Rio, 1986, 6 p. (Comunicado Técnico, 174). 